\title{
Gate-controlled ionization and screening of cobalt adatoms on a graphene surface
}

\author{
Victor W. Brarr, ${ }^{1,2 \star \dagger}$, Régis Decker ${ }^{1,2 \dagger}$, Hans-Michael Solowan ${ }^{1 \dagger}$, Yang Wang ${ }^{1}$, Lorenzo Maserati ${ }^{1}$, \\ Kevin T. Chan ${ }^{1,2}$, Hoonkyung Lee ${ }^{1,2}$, Çağlar O. Girit ${ }^{1,2}$, Alex Zettt ${ }^{1,2}$, Steven G. Louie ${ }^{1,2}$, \\ Marvin L. Cohen ${ }^{1,2}$ and Michael F. Crommie ${ }^{1,2}$
}

\begin{abstract}
Graphene impurities provide both a source of mobility-limiting disorder and a means to desirably alter graphene electronic structure. Adsorbates on graphene can, for example, induce Coulomb scattering ${ }^{1,2}$, alter electron-phonon interactions ${ }^{3}$, shift the chemical potential ${ }^{1,2}$, change the effective dielectric constant ${ }^{4}$ and-in cases such as 'graphane ${ }^{15}$ - form whole new two-dimensional materials. While these effects have thus far been primarily studied with spatially averaged techniques, understanding the microscopic physics of such behaviour requires local-probe exploration of the subnanometre-scale electronic and structural properties of impurities on graphene. Here we describe scanning tunnelling microscopy and spectroscopy measurements made on individual Co atoms deposited onto back-gated graphene devices. We find that the electronic structure of Co adatoms can be tuned by application of the device gate voltage, and that the Co atoms can be reversibly ionized. Large screening clouds are observed to form around Co adatoms ionized in this way, and we observe that some intrinsic graphene defects also show charging behaviour. Our results provide new insight into charged-impurity scattering in graphene, as well as the possibility of using graphene devices as chemical sensors.
\end{abstract}

Our samples were prepared by evaporating Co atoms onto a back-gated graphene/ $\mathrm{SiO}_{2}$ device held inside the scanning tunnelling microscope at $4.2 \mathrm{~K}$ (see Methods). Figure 1 shows a representative $10 \times 10 \mathrm{~nm}^{2}$ scanning tunnelling microscopy (STM) image of a graphene surface following Co adatom deposition. Co adatoms appear as $\sim 4$ - $\AA$-high dome-like protrusions on the graphene surface (two cobalt adatoms can be seen in Fig. 1).

We made differential conductance, $\mathrm{d} I / \mathrm{d} V$, measurements with the STM tip held over individual Co adatoms to measure their energy-dependent local density of states (LDOS). Figure 2a shows typical $\mathrm{d} I / \mathrm{d} V$ spectra obtained from a single Co adatom for different applied back-gate voltages, $V_{\mathrm{G}}$ (changing $V_{\mathrm{G}}$ changes the graphene charge-carrier density). Three different types of feature are observed in these spectra. The first is a dip observed at the Fermi level $\left(E_{\mathrm{F}}\right)$ with a full-width at half-maximum of $\sim 10 \mathrm{meV}$ (see the inset for a higher-resolution spectrum). The energy and width of this feature does not vary significantly with $V_{\mathrm{G}}$. The second feature is a series of resonant peaks in the $\mathrm{d} I / \mathrm{d} V$ signal marked A, B, C and D in Fig. 2a. These peaks have a typical full-width at half-maximum of $25 \pm 5 \mathrm{meV}$. Their behaviour differs from the dip at $E_{\mathrm{F}}$ in that their energy locations move in the same direction (and by similar amounts) as the Dirac point voltage $\left(V_{\mathrm{D}}\right)$ as $V_{\mathrm{D}}$ is varied by application of $V_{\mathrm{G}}\left(V_{\mathrm{D}}\right.$ marks the location of a dip seen

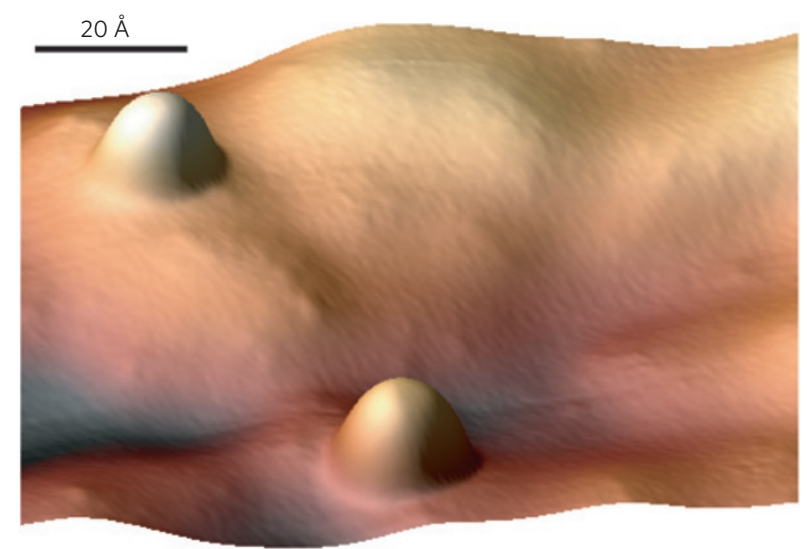

0

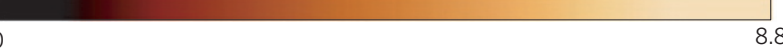

(

Figure 1 | $10 \times 10 \mathrm{~nm}^{2}$ STM topograph showing two cobalt adatoms resting atop a graphene sheet on a $\mathrm{SiO}_{2}$ substrate (tunnelling parameters: $V_{b}=-0.25 \mathrm{~V}, I=10 \mathrm{pA}, V_{\mathrm{G}}=0 \mathrm{~V}$ ).

in the graphene LDOS which is associated with the Dirac point energy $\left.^{6}\right)$. The third feature corresponds to the peak in the $\mathrm{d} I / \mathrm{d} V$ signal marked $\mathrm{S}$ in Fig. 2a. The energy of this feature changes as $V_{\mathrm{G}}$ is varied, but it shifts energetically in the opposite direction compared with $V_{\mathrm{D}}$ and disperses significantly faster. Figure 2a focuses only on spectra measured for $-60 \mathrm{~V}<V_{\mathrm{G}}<-20 \mathrm{~V}$ because all dispersive features $(\mathrm{A}, \mathrm{B}, \mathrm{C}, \mathrm{D}, \mathrm{S})$ shift out of the measurement window $\left(-0.15 \mathrm{~V}<V_{b}<+0.15 \mathrm{~V} ; V_{b}\right.$ is the voltage of the sample with respect to the tip) for $-20 \mathrm{~V}<V_{\mathrm{G}}<+60 \mathrm{~V}$ (see Supplementary Information for measurements of $-20 \mathrm{~V}<V_{\mathrm{G}}<+60 \mathrm{~V}$ ). Figure $2 \mathrm{~b}$ summarizes how these spectral features shift in energy as a function of $V_{\mathrm{G}}$. Also plotted in Fig. $2 \mathrm{~b}$ is the gate-dependent variation in Dirac point voltage $\left(V_{\mathrm{D}}\right)$ measured on the graphene surface at least $10 \mathrm{~nm}$ away from any Co atom.

To better understand how the presence of a Co adatom affects the behaviour of graphene, $\mathrm{d} I / \mathrm{d} V$ measurements were also made on the bare graphene surface adjacent to a Co adatom. Figure $3 \mathrm{a}$ shows $\mathrm{d} I / \mathrm{d} V\left(V_{b}\right)$ and $I\left(V_{b}\right)$ curves measured on graphene $\sim 2.5 \mathrm{~nm}$ away (laterally) from the centre of a Co adatom. The $\mathrm{d} I / \mathrm{d} V$ spectrum shows a $\sim 126$-meV-wide gap-like feature at $E_{\mathrm{F}}$ as expected for clean graphene (this is known to arise from inelastic

${ }^{1}$ Department of Physics, University of California at Berkeley, Berkeley, California 94720, USA, ${ }^{2}$ Materials Sciences Division, Lawrence Berkeley Laboratory, Berkeley, California 94720, USA. ${ }^{\dagger} T h e s e$ authors contributed equally to this work. *e-mail: vbrar@berkeley.edu. 
a

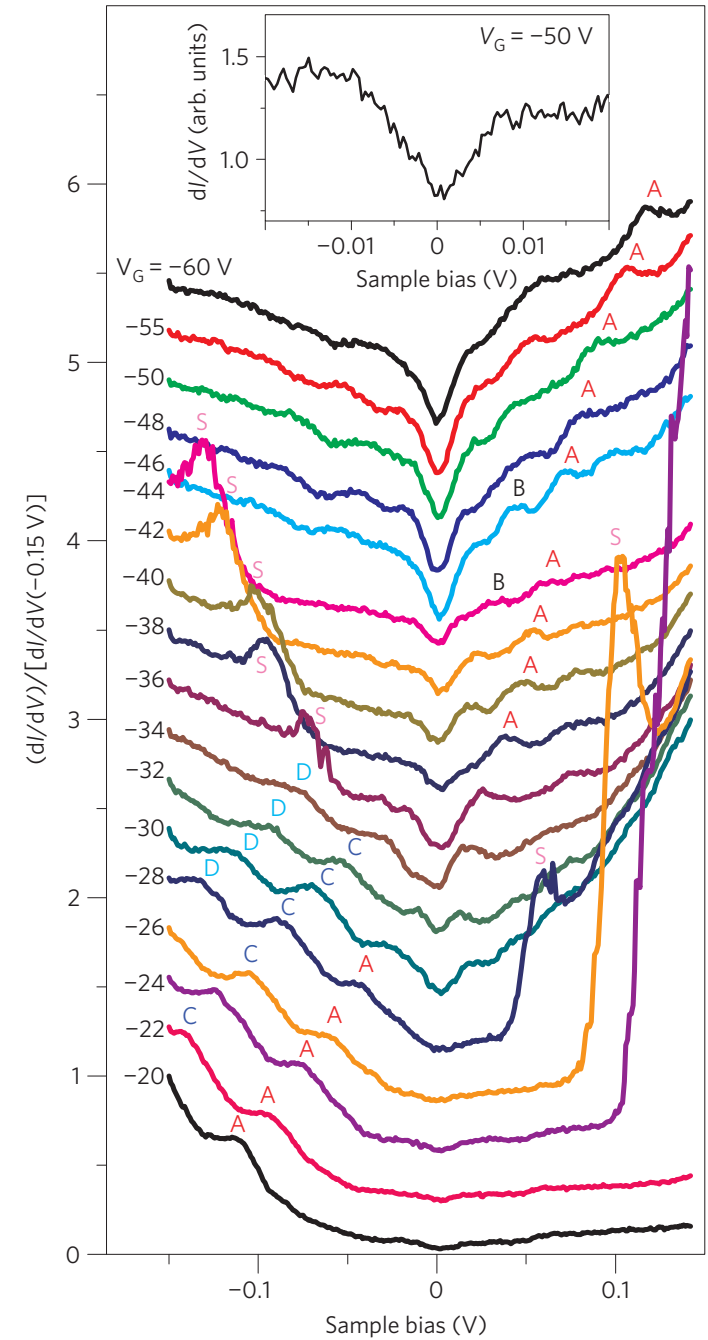

b

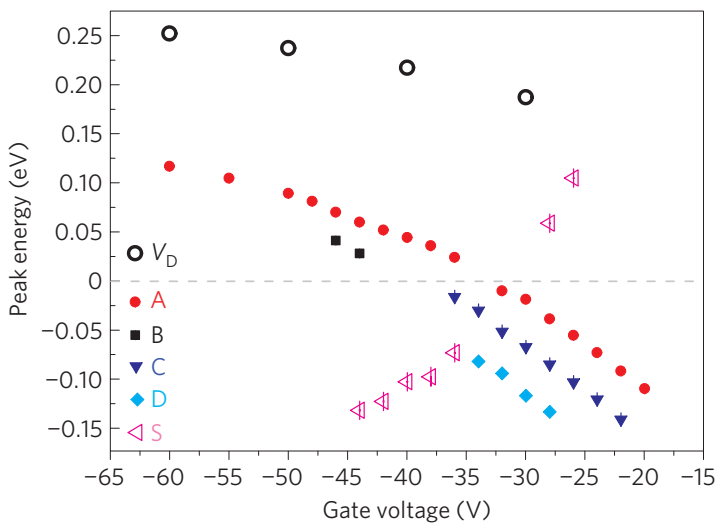

Figure 2 | Gate-dependent $\mathrm{d} / \mathrm{d} V$ spectra of Co adatom on graphene. a, $\mathrm{d} / \mathrm{d} V$ spectra taken with the tip directly above a cobalt adatom on graphene for different back-gate voltages $\left(V_{G}\right)$ (initial tunnelling parameters: $V_{b}=+0.15 \mathrm{~V}, I=22 \mathrm{pA}$; wiggle voltage $V_{r m s}=4 \mathrm{mV}$ ). Carrier-density-dependent spectral features are labelled $A, B, C, D$ and $S$. Inset: High-resolution $\mathrm{d} / \mathrm{d} V$ spectrum of the dip-like feature at the Fermi level (initial tunnelling parameters: $V_{b}=-0.08 \mathrm{~V}, I=8 \mathrm{pA}, V_{\mathrm{G}}=-50 \mathrm{~V}$; wiggle voltage $V_{\mathrm{rms}}=1 \mathrm{mV}$ ). $\mathbf{b}$, Gate-voltage dependence of $\mathrm{d} / / \mathrm{d} V$ spectral features observed in $\mathbf{a}$. The error bars represent the uncertainty in the Lorentzian fitting of each spectral feature after a linear background subtraction. The open circles indicate the Dirac point voltage $\left(V_{D}\right)$ measured $10 \mathrm{~nm}$ from the cobalt atom. tunnelling ${ }^{6}$ ), but an extra sharp dip is also observed $\sim 220 \mathrm{meV}$ above $E_{\mathrm{F}}$. We measured how this new $\mathrm{d} I / \mathrm{d} V$ feature varies spatially through the use of $\mathrm{d} I / \mathrm{d} V$ mapping. Figure $3 \mathrm{~b}-\mathrm{d}$ shows $\mathrm{d} I / \mathrm{d} V$ maps acquired near a single Co atom with different $V_{\mathrm{G}}$ and $V_{b}$. The most striking aspect of these images is a narrow ring, arising from the spectral dip feature above $E_{\mathrm{F}}$, that appears centred around the Co adatom. The diameter of this ring is strongly dependent on $V_{b}$ and $V_{\mathrm{G}}$. For example, Fig. $3 \mathrm{c}$,d shows the ring diameter increasing as $V_{\mathrm{G}}$ decreases, and Fig. 3b,d shows the diameter increasing as $V_{b}$ increases.

$\mathrm{d} I / \mathrm{d} V$ maps were also acquired over the bare graphene surface (that is, in the absence of cobalt adatoms) after annealing to higher temperatures $\left(400-500^{\circ} \mathrm{C}\right)$, as seen in Fig. 3e. Ring-like features similar to those found around Co adatoms are visible. The inset of Fig. 3f shows an STM topograph of the centre of one of these rings, revealing a defect in the graphene. $\mathrm{A} d I / \mathrm{d} V$ spectrum taken at the site of such a defect (Fig. 3f) shows two clear spectroscopic features, $R^{\prime}$ and $S^{\prime} . R^{\prime}$ is observed to disperse in the same direction as $V_{\mathrm{D}}$ when $V_{\mathrm{G}}$ is varied, whereas $\mathrm{S}^{\prime}$ disperses in the opposite direction. The gate-voltage dependence of $\mathrm{R}^{\prime}$ is thus similar to that seen for the Co resonant states $\mathrm{A}, \mathrm{B}, \mathrm{C}$ and $\mathrm{D}$, whereas the gate dependence of $\mathrm{S}^{\prime}$ is similar to the Co $S$ feature. For fixed $V_{\mathrm{G}}$, however, the energies of $\mathrm{R}^{\prime}$ and $\mathrm{S}^{\prime}$ (seen for numerous defects) differ from the characteristic energies of the spectral features observed for Co adatoms.

The spectroscopic features we observed for cobalt adatoms and intrinsic graphene defects can be understood within a general impurity-physics framework, which we now describe. We start with the dip feature at $E_{\mathrm{F}}$ observed for cobalt adatoms. This is not a conventional band-structure feature because it remains pinned to $E_{\mathrm{F}}$ even as $E_{\mathrm{F}}$ is swept through the graphene band-structure by back-gating. One possible origin of this feature is the Kondo effect, which can lead to a resonance at $E_{\mathrm{F}}$ due to spin-screening of a local moment ${ }^{7}$. While cobalt is expected to be magnetic on graphene ${ }^{8-10}$, a Kondo resonance is generally expected to have a strongly gate-dependent width ${ }^{11}$, which is not observed here. This opens the possibility that the dip at $E_{\mathrm{F}}$ arises from another origin, such as vibrational inelastic electron tunnelling ${ }^{12}$. Inelastic electron tunnelling causes an increase in $\mathrm{d} I / \mathrm{d} V$ conductance at vibrational-energy thresholds for both positive and negative biases, and so can produce dip-like features in $\mathrm{d} I / \mathrm{d} V$ spectra with a half-width equal to the vibrational mode energy ${ }^{12}$. To test this latter possibility, we carried out $a b$ initio calculations of the vibrational energies of a Co adatom on graphene (see Methods). Our simulation indicates that this system has in-plane vibrational modes of 12 and $27 \mathrm{meV}$, as well as out-of-plane modes of 17, 40 and $53 \mathrm{meV}$. The lowest-energy mode calculated here is similar in magnitude to the $5 \mathrm{meV}$ half-width of the dip we observe for cobalt adatoms, suggesting a possible inelastic electron tunnelling origin for this experimental feature.

We now turn to the cobalt-induced resonance features marked A, B, C and D in Fig. 2a,b. We identify these features with the impurity DOS of the combined cobalt/graphene electronic structure because they shift energetically in the same direction as the Dirac point voltage $\left(V_{\mathrm{D}}\right)$ as $E_{\mathrm{F}}$ is swept through the graphene bandstructure. Figure $4 a(i), b(i)$ shows sketches of how an impurityinduced DOS feature can be expected to shift as a result of applied gate bias. A likely explanation for these resonances (owing to their narrow energy width and spatial localization) is that they arise from a hybridization of cobalt atomic levels with graphene continuum states. Such resonances have been predicted for transition-metal atoms on graphene $e^{9,10,13}$, but our observed energy-level spacings and multiplicity do not precisely match these predictions. A possible explanation for the multiplicity of modes is hybrid electronicvibrational (that is, vibronic) ${ }^{14}$ states, which are expected to have an energy spacing equal to the energy of a vibrational mode (our experimentally observed energy spacing is on the order of the 

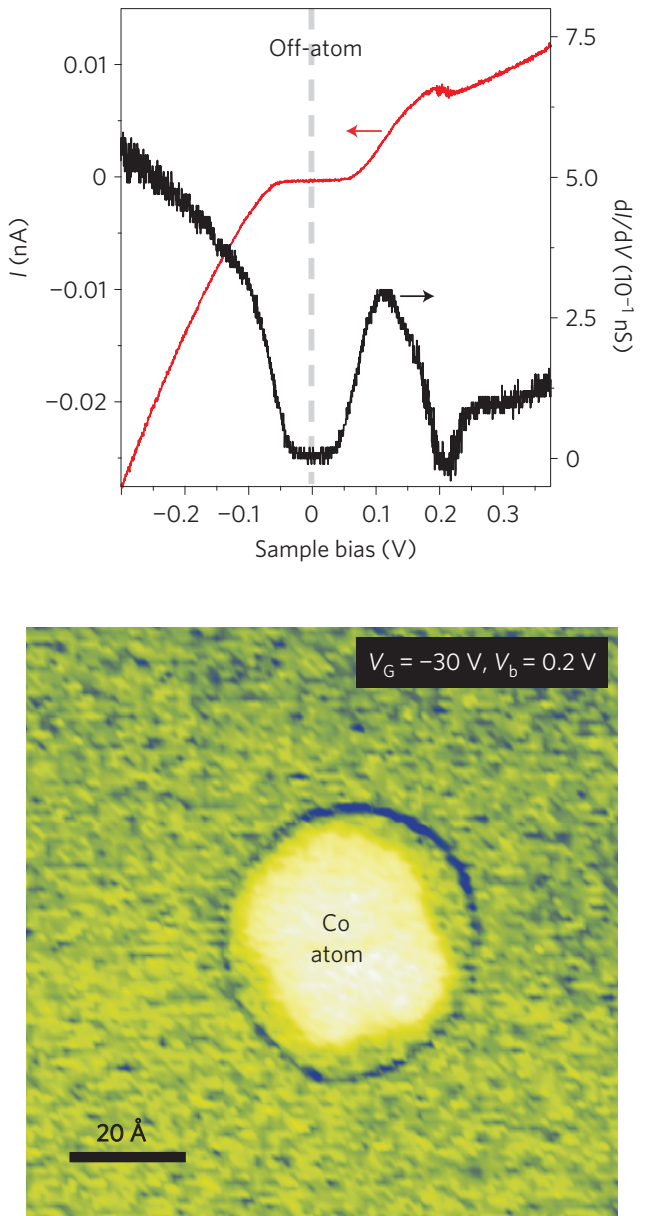

e

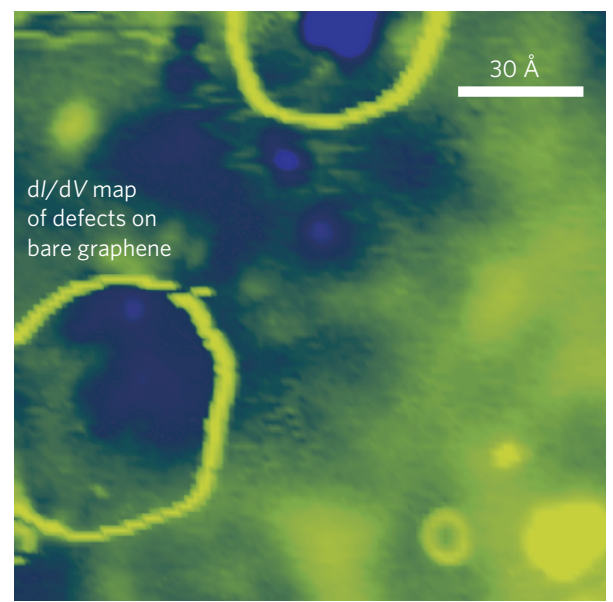

b

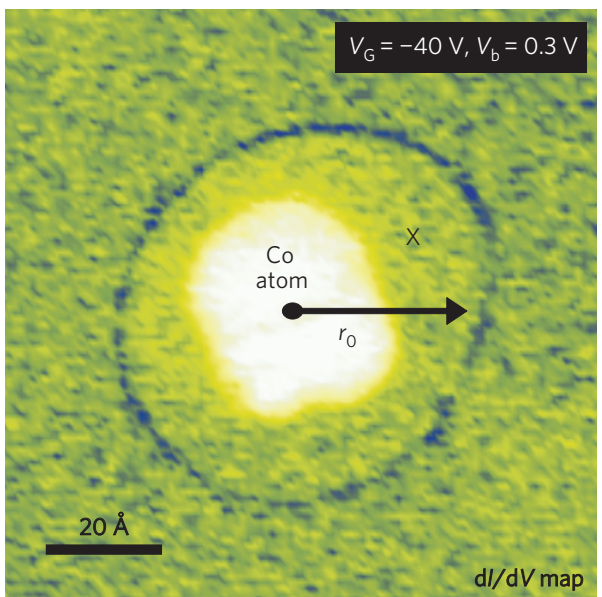

d

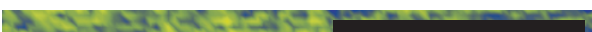

f

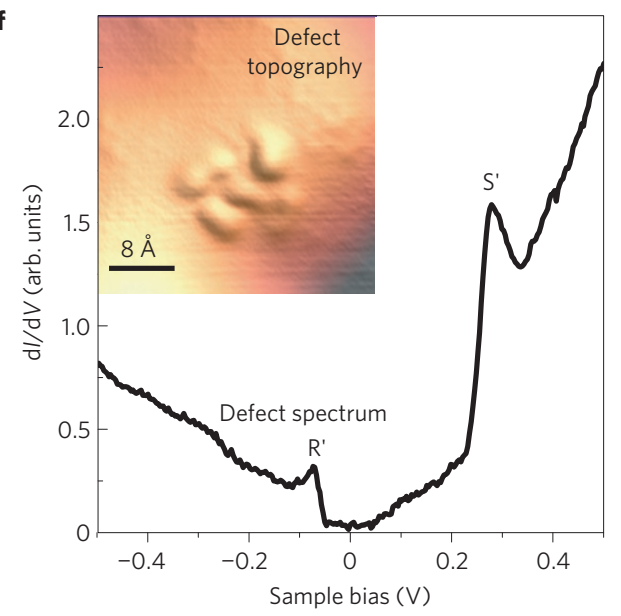

Figure $3 \mid \mathrm{d} l / \mathrm{d} V$ conductance measurements of the graphene surface near Co adatoms and surface defects. a, Off-atom $d l / d V\left(V_{b}\right)$ and $I\left(V_{b}\right)$ spectra of

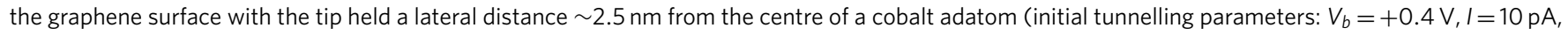
$V_{G}=-40 \mathrm{~V}$ ). b-d, $d / d \mathrm{~d} V$ maps of a single cobalt adatom and the surrounding graphene surface for varying $V_{b}$ and $V_{G}$ values. The ' $X$ ' in $\mathbf{b}$ indicates the


in vacuum. Observed ring-like features are centred on defects in the graphene (tunnelling parameters: $V_{b}=+0.7 \mathrm{~V}, I=8 \mathrm{pA}, V_{\mathrm{G}}=-4 \mathrm{~V}$ ). $\mathbf{f}, \mathrm{d} l / \mathrm{d} V$ spectrum taken with the tip held over the defect site found at the centre of a defect-induced ring such as those seen in e. Two prominent features are

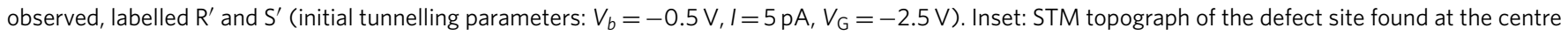
of a ring-like feature similar to those shown in e (tunnelling parameters: $V_{b}=-0.3 \mathrm{~V}, I=10 \mathrm{pA}, V_{\mathrm{G}}=-60 \mathrm{~V}$ ).

energies we calculated for the out-of-plane Co/graphene vibrational modes). Finally, we point out that these states might be related to predicted fluctuations in LDOS due to screening of a 'supercritical' Coulomb impurity on graphene (that is, quasi-Rydberg states predicted in an 'atomic-collapse' scenario) ${ }^{15,16}$.
Regardless of their origin, the Co-atom DOS features can be either emptied or filled with electrons as they are moved above or below $E_{\mathrm{F}}$ through application of a gate voltage. The energy position of these resonances with respect to $E_{\mathrm{F}}$ determines the ionization state of a Co atom, and the Co ionization state can thus be externally 
a

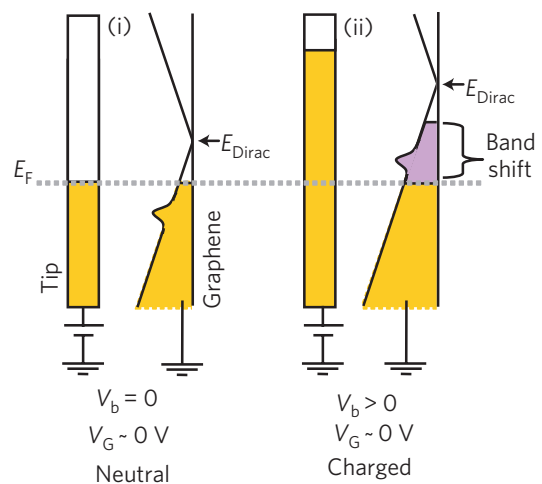

b
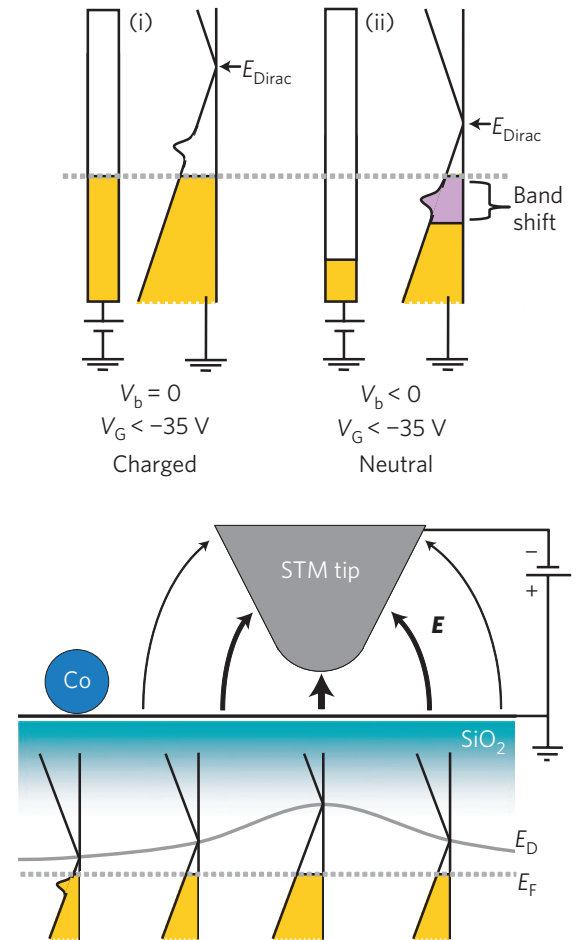

Figure 4 | Schematic diagram of the tip and back-gate-induced ionization of an adatom on graphene. $\mathbf{a}(\mathbf{i})$, For $V_{b}=0$ and $V_{G} \sim 0 V$ the adatom impurity DOS feature (that is, resonance) is below $E_{F}$ and the adatom is neutral. a(ii), When a negative voltage is applied to the STM tip $\left(V_{b}>0\right)$ the graphene bands shift upwards relative to $E_{F}$ (that is, there is a reduction in the local electron density of the graphene). A large enough shift pulls the impurity state above $E_{F}$, thus changing the adatom charge state. $\mathbf{b}(\mathbf{i})$, For $V_{b}=0$ and $V_{G}<-35 V$ the Co impurity state is above $E_{F}$ and the Co adatom is charged (ionized). $\mathbf{b}$ (ii), When a positive bias is applied to the STM tip $\left(V_{b}<0\right)$ the graphene bands shift downwards relative to $E_{F}$. A large enough shift will push the impurity state below $E_{F}$, thus returning the cobalt atom to a neutral state. c, Schematic diagram showing the positional dependence of the band-bending induced by an STM tip (considering $V_{b}$ and work-function differences). In this figure the Co adatom is neutral, and the gating effect due to the tip at the position of the Co atom is less than in $\mathbf{a}$ and $\mathbf{b}$, where the tip was considered to be directly above the adatom (see Supplementary Information).

controlled using a gate voltage. This interpretation is supported by the existence of both the $S$ state and the ring structure surrounding each atom. We first discuss the $S$ state, which moves in the opposite direction in energy compared with both $V_{\mathrm{D}}$ and the resonant peaks when a gate voltage is applied (Fig. 2a,b). This behaviour is the reverse of what is expected for a typical DOS feature, but makes sense in the context of Co-atom ionization ${ }^{17}$. Here the application of a tip bias (as well as any difference in tip-sample work functions) causes a local gating of the sample under the tip, which is added to the more global gating caused by the back-gate electrode, thus inducing the impurity DOS to rise $\left(V_{b}>0\right)$ or fall $\left(V_{b}<0\right)$ with respect to $E_{\mathrm{F}}$. The Co atom becomes ionized when the tip bias is great enough to cause impurity states to cross $E_{\mathrm{F}}$, thus creating a screening-induced response in the $\mathrm{d} I / \mathrm{d} V$ signal (that is, the $S$ peak). As shown in Fig. 4, this mechanism works equally well in the case when the impurity state is placed above $E_{\mathrm{F}}$ by the back-gate $\left(V_{\mathrm{G}}<-35 \mathrm{~V}\right)$ and when the impurity state is held below $E_{\mathrm{F}}\left(V_{\mathrm{G}}>-35 \mathrm{~V}\right)$, except that the $\mathrm{S}$ peak is seen on opposite sides of $E_{\mathrm{F}}$ for these two cases. Such behaviour is seen in the data of Fig. 2a,b, where the resonant peaks and $\mathrm{S}$ state lie on opposite sides of $E_{\mathrm{F}}$ and (in the case of state A) even cross $E_{\mathrm{F}}$ at the same $V_{\mathrm{G}}$. This ionization framework also explains the behaviour of the $\mathrm{R}^{\prime}$ and $\mathrm{S}^{\prime}$ resonances seen for graphene defects (the $\mathrm{R}^{\prime}$ and $S^{\prime}$ defect features play the same role as the cobalt $A$ and $S$ features, respectively).

Such ionization behaviour can be quantitatively analysed for our data using a simple double-gate model (that is, graphene plus back-gate plus tip-gate). Here we fix the back-gate voltage $\left(V_{\mathrm{G}}\right)$ and calculate the local band-bending arising from the electric potential difference (as well as the work-function difference) between the STM tip and the graphene substrate beneath it (that is, we calculate the change in the local graphene electronic density due to tipsample capacitance $)^{18,19}$. For the case of $V_{\mathrm{G}}=-40 \mathrm{~V}$ we calculate that an applied potential difference of $V_{b}=-105 \mathrm{mV}$ will cause impurity ionization for states observed at $V_{b}=+45 \mathrm{mV}$ or less (see Methods and Supplementary Information). This correlates well with our spectroscopy of Co adatoms for $V_{\mathrm{G}}=-40 \mathrm{~V}$, where we observe an impurity state (A) at $V_{b}=+44 \mathrm{mV}$ and an ionization peak $(\mathrm{S})$ at $V_{b}=-100 \mathrm{mV}$.

The gate-dependent ionization of cobalt atoms also explains the ring structure seen surrounding each atom in $\mathrm{d} I / \mathrm{d} V$ maps and topography (Fig. 3). As shown in Fig. 4c, when the tip is displaced laterally from an atom its electric field can still induce a local gating that shifts cobalt impurity states with respect to $E_{\mathrm{F}}$, causing ionization (such tip-induced gating has been previously observed in different physical systems $\left.{ }^{17,20,21}\right)$. This ionization leads to a change in graphene LDOS surrounding the atom arising from screening charge, and a resulting change in the measured $\mathrm{d} I / \mathrm{d} V$ at the radius, $r_{0}$, where the ionization takes place. The cobalt atom is ionized when the tip is held at a lateral distance $r<r_{0}$ for such a bias and gate voltage (that is, tip inside the ring), whereas for $r>r_{0}$ the atom is not ionized (that is, tip outside the ring). The size of $r_{0}$ is dependent on $V_{b}, V_{\mathrm{G}}$, tip height and tip shape. For example, if $V_{\mathrm{G}}$ is changed such that the impurity DOS features are further from $E_{\mathrm{F}}$, then the STM tip will need to be brought closer to the Co adatom to ionize it. This expected behaviour corresponds to the experimental behaviour shown in Fig. 3b-d. This type of behaviour also explains the appearance of ring structures around graphene defects, as seen in Fig. 3e,f, indicating that defects can induce localized states (as predicted theoretically ${ }^{22,23}$ ) that exhibit gate-induced charging/discharging. Tip-induced ionization rings have previously been seen in systems exhibiting an energy gap (unlike graphene) such as impurities on $\mathrm{C}_{60}$ films ${ }^{17}$ and dopants in semiconductor systems ${ }^{20,21}$.

The rings we observe in graphene around cobalt atoms are indicative of a screening cloud that surrounds each charged adatom. We have observed rings with diameters greater than $12 \mathrm{~nm}$ (for $V_{\mathrm{G}}<-50 \mathrm{~V}$ ), indicating that the screening clouds can be quite large and will probably affect macroscopic transport measurements (charged impurity scattering in general has already been proposed as a main limitation of graphene mobility $\left.{ }^{1,24}\right)$. The positioning of localized graphene impurity and defect states either above or below the Dirac point can thus explain asymmetries in graphene electron and hole conduction observed in recent transport measurements ${ }^{1,2}$. Although often viewed as a problem, 
such behaviour could potentially be used in a graphene chemical sensor. In this scenario, changes in the graphene conductivity at particular back-gate voltages could be correlated with the ionization thresholds of particular chemical species.

\section{Methods}

Our experiments were carried out using an Omicron LT-STM operating in ultrahigh vacuum $\left(<10^{-11}\right.$ torr) and at $4.2 \mathrm{~K}$. Scanning tunnelling microscope tips were calibrated spectroscopically against the $\mathrm{Au}(111)$ Shockley surface state before all measurements. STM differential conductance $(\mathrm{d} I / \mathrm{d} V)$ was measured by lock-in detection of the a.c. tunnel current modulated by a $1-5 \mathrm{mV}(\mathrm{rms})$, $350-500 \mathrm{~Hz}$ signal added to the tunnelling bias $V_{b}$. The $\mathrm{d} I / \mathrm{d} V\left(V_{b}\right)$ and $I\left(V_{b}\right)$ measurements were made under open feedback conditions with the tip in a fixed position. Single graphene sheets were prepared using either mechanical exfoliation $^{25}$ or by chemical vapour deposition ${ }^{26}$. In both cases the graphene sheets were placed on a 285-nm-thick $\mathrm{SiO}_{2}$ layer grown atop a heavily doped $\mathrm{Si}$ crystal, which was used as a back-gate electrode ${ }^{25}$. Electrical contact was made to the graphene by direct deposition of Ti (10-nm-thick)/Au (30-nm-thick) electrodes through a stencil mask. Samples were annealed at temperatures between $200^{\circ} \mathrm{C}$ and $400^{\circ} \mathrm{C}$ for several hours in ultrahigh vacuum to clean them before cooling. Co adatoms were deposited by e-beam evaporation onto pristine, cold $(<10 \mathrm{~K})$ graphene surfaces showing large areas (for example, $\left.20 \times 20 \mathrm{~nm}^{2}\right)$ with no observable defects.

These measurements were reproduced on more than 50 Co adatoms measured with ten different PtIr tips on five different graphene devices (three exfoliated, two chemical vapour deposition grown). Although the majority of atoms measured in this work showed the behaviour described here, a fraction $(\sim 30 \%)$ of atom-like protrusions observed in our measurements strayed from this behaviour in non-systematic ways. We attribute these either to Co dimers and trimers that formed as a result of diffusion during evaporation, or to Co adatoms that have attached to defects in the graphene surface. To test for atomic-hydrogen contamination in our system (which can lead to dip-like features at the Fermi energy ${ }^{27}$ ), we also evaporated Co atoms onto a $\mathrm{Cu}(111)$ surface. Subsequent $\mathrm{d} I / \mathrm{d} V$ measurements of $50 \mathrm{Co} / \mathrm{Cu}(111)$ adatoms showed the typical $\sim 5-\mathrm{mV}$-wide Kondo feature expected for this system. None of the height abnormalities or spectroscopic artefacts related to hydrogen contamination ${ }^{27}$ were observed.

To understand the possible role of defects in our measurements, defects were introduced into some graphene surfaces through high-temperature annealing $\left(400-500^{\circ} \mathrm{C}\right)$ (a topographic image of a typical defect can be seen in Fig. 3f, inset). We observe that the presence of nearby defects did not change the behaviour of subsequently deposited Co adatoms. Furthermore, atomic manipulation was used to insure that Co adatoms studied were not bound to defect sites.

Our ab initio calculations of the vibrational modes of a Co adatom on graphene were carried out with the Quantum-Espresso $\operatorname{code}^{28}$ using density functional theory within the generalized gradient approximation ${ }^{29}$. Ultrasoft pseudopotentials and a plane-wave basis set were used. Kinetic-energy cutoffs of $45 \mathrm{Ry}$ and $360 \mathrm{Ry}$ were used for the wavefunctions and charge density, respectively. The system was modelled with a $4 \times 4$ graphene supercell containing $32 \mathrm{C}$ atoms and one Co atom on a hollow site. Vibrational modes were calculated using density functional perturbation theory ${ }^{30}$.

In our calculation of the ionization threshold voltage of a Co adatom we roughly modelled the tip as a flat electrode with a work function $\Phi_{\text {tip }}=4.8 \mathrm{eV}$ and held $6 \AA$ above the graphene surface. The actual tip starts as an electrochemically etched PtIr wire, but undergoes some interaction with a gold surface, so the final shape and composition (and work function) are uncertain. Additionally, there is always uncertainty in the precise distance of an STM tip from a surface. While our model provides a good framework for understanding the data, the accuracy is limited at present because the expected band-bending is a nonlinear function of $V_{\mathrm{G}}, V_{b}$, work-function difference and tip height. Changing parameters such as $\Phi_{\text {tip }}$ or the tip height by $\sim 30 \%$ could easily change the calculated threshold of ionization by a factor of two (see Supplementary Information).

\section{Received 21 May 2010; accepted 6 September 2010;} published online 31 October 2010

\section{References}

1. Chen, J. H. et al. Charged-impurity scattering in graphene. Nature Phys. 4 377-381 (2008).

2. Pi, K. et al. Electronic doping and scattering by transition metals on graphene. Phys. Rev. B 80, 075406 (2009).

3. McChesney, J. L. et al. Extended van Hove singularity and superconducting instability in doped graphene. Phys. Rev. Lett. 104, 136803.

4. Jang, C. et al. Tuning the effective fine structure constant in graphene: Opposing effects of dielectric screening on short- and long-range potential scattering. Phys. Rev. Lett. 101, 146805 (2008).

5. Elias, D. C. et al. Control of graphene's properties by reversible hydrogenation: Evidence for graphane. Science 323, 610-613 (2009).
6. Zhang, Y. et al. Giant phonon-induced conductance in scanning tunnelling spectroscopy of gate-tunable graphene. Nature Phys. 4, 627-630 (2008).

7. Kondo, J. Resistance minimum in dilute magnetic alloys. Prog. Theor. Phys. 32, 37-49 (1964).

8. Uchoa, B., Kotov, V. N., Peres, N. M. R. \& Castro Neto, A. H. Localized magnetic states in graphene. Phys. Rev. Lett. 101, 026805 (2008).

9. Wehling, T. O., Balatsky, A. V., Katsnelson, M. I., Lichtenstein, A. I. \& Rosch, A. Orbitally controlled Kondo effect of Co adatoms on graphene. Phys. Rev. B 81, 115427 (2010).

10. Yuliang, M. et al. Density functional calculation of transition metal adatom adsorption on graphene. J. Phys. Condens. Matter 20, 115209 (2008).

11. Goldhaber-Gordon, D. et al. From the Kondo regime to the mixed-valence regime in a single-electron transistor. Phys. Rev. Lett. 81, 5225-5228 (1998).

12. Stipe, B. C., Rezaei, M. A. \& Ho, W. Single-molecule vibrational spectroscopy and microscopy. Science 280, 1732-1735 (1998).

13. Chan, K. T., Neaton, J. B. \& Cohen, M. L. First-principles study of metal adatom adsorption of graphene. Phys. Rev. B 77, 235430 (2008).

14. Qiu, X. H., Nazin, G. V. \& Ho, W. Vibronic states in single molecule electron transport. Phys. Rev. Lett. 92, 206102 (2004)

15. Shytov, A. V., Katsnelson, M. I. \& Levitov, L. S. Atomic collapse and quasi-Rydberg states in graphene. Phys. Rev. Lett. 99, 246802 (2007).

16. Pereira, V. M., Nilsson, J. \& Castro Neto, A. H. Coulomb impurity problem in graphene. Phys. Rev. Lett. 99, 166802 (2007).

17. Pradhan, N. A., Liu, N., Silien, C. \& Ho, W. Atomic scale conductance induced by single impurity charging. Phys. Rev. Lett. 94, 076801 (2005).

18. Luryi, S. Quantum capacitance devices. Appl. Phys. Lett. 52, 501-503 (1988)

19. Feenstra, R. M. \& Stroscio, J. A. Tunneling spectroscopy of the GaAs(110) surface. J. Vac. Sci. Technol. B 5, 923-929 (1987).

20. Marczinowski, F., Wiebe, J., Meier, F., Hashimoto, K. \& Wiesendanger, R. Effect of charge manipulation on scanning tunnelling spectra of single $\mathrm{Mn}$ acceptors in InAs. Phys. Rev. B 77, 115318 (2008).

21. Teichmann, K. et al. Controlled charge switching on a single donor with a scanning tunneling microscope. Phys. Rev. Lett. 101, 076103 (2008).

22. Pereira, V. M., Guinea, F., Lopes dos Santos, J. M. B., Peres, N. M. R. \& Castro Neto, A. H. Disorder induced localized states in graphene. Phys. Rev. Lett. 96, 036801 (2006).

23. Wehling, T. O. et al. Local electronic signatures of impurity states in graphene. Phys. Rev. B 75, 125425 (2007)

24. Adam, S., Hwang, E. H., Galitski, V. M. \& Das Sarma, S. A self-consistent theory for graphene transport. Proc. Natl Acad. Sci. 104, 18392-18397 (2007).

25. Novoselov, K. S. et al. Electric field effect in atomically thin carbon films. Science 306, 666-669 (2004).

26. Li, X. et al. Large-area synthesis of high-quality and uniform graphene films on copper foils. Science 324, 1312-1314 (2009).

27. Markus, T. et al. Spectroscopic manifestations of the Kondo effect on single adatoms. J. Phys. Condens. Matter 21, 053001 (2009).

28. Paolo, G. et al. QUANTUM ESPRESSO: A modular and open-source software project for quantum simulations of materials. J. Phys. Condens. Matter 21, 395502 (2009).

29. Perdew, J. P., Burke, K. \& Ernzerhof, M. Generalized gradient approximation made simple. Phys. Rev. Lett. 77, 3865-3868 (1996).

30. Baroni, S., de Gironcoli, S., Dal Corso, A. \& Giannozzi, P. Phonons and related crystal properties from density-functional perturbation theory. Rev. Mod. Phys. 73, 515-562 (2001).

\section{Acknowledgements}

We thank J. Repp for discussions. The research was supported by the Director, Office of Science, Office of Basic Energy Sciences, Materials Sciences and Engineering Division, of the US Department of Energy under contract No DE-AC02-05CH11231 (STM-instrumentation development and operation), by the Office of Naval Research MURI Award No N00014-09-1-1066 (experimental-data analysis) and by National Science Foundation grant Nos DMR-0906539 (graphene-device synthesis) and DMR-0705941 (electronic-structure calculation). Computational resources have been provided by DOE at Lawrence Berkeley National Laboratory's NERSC facility and the Lawrencium computational cluster resource provided by the IT Division at the Lawrence Berkeley National Laboratory. H-M.S. was partially supported by the German Academic Exchange Service. R.D. acknowledges a postdoctoral fellowship from the Swiss National Science Foundation.

\section{Author contributions}

V.W.B., R.D., H-M.S. and M.F.C. designed the experiment and made the measurements. V.W.B., R.D., H-M.S., Y.W., K.T.C., H.L. and M.F.C. carried out the analysis. Y.W., L.M., C.O.G. and A.Z. facilitated the sample fabrication. K.T.C., H.L., S.G.L. and M.L.C. carried out ab initio calculations. V.W.B. and M.F.C. wrote the manuscript.

\section{Additional information}

The authors declare no competing financial interests. Supplementary information accompanies this paper on www.nature.com/naturephysics. Reprints and permissions information is available online at http://npg.nature.com/reprintsandpermissions. Correspondence and requests for materials should be addressed to V.W.B. 